\title{
A class of transcendental numbers with explicit $g$-adic expansion and the Jacobi-Perron algorithm
}

by

Jun-ICHi TAmura (Tokyo)

1. Introduction. In this paper, we give transcendental numbers $\varphi$ and $\psi$ such that

(i) both $\varphi$ and $\psi$ have explicit $g$-adic expansions, and simultaneously,

(ii) the vector ${ }^{t}(\varphi, \psi)$ has an explicit expression in the Jacobi-Perron algorithm (cf. Theorem 1).

Our results can be regarded as a higher-dimensional version of some of the results in [1]-[5] (see also [6]-[8], [10], [11]). The numbers $\varphi$ and $\psi$ have some connection with algebraic numbers with minimal polynomials $x^{3}-k x^{2}-l x-1$ satisfying

$$
k \geq l \geq 0, \quad k+l \geq 2 \quad(k, l \in \mathbb{Z}) .
$$

In the special case $k=l=1$, our Theorems $1-3$ have been shown in [15] by a different method using the theory of representation of numbers by Fibonacci numbers of third degree.

2. Notations and main results. We begin with some notations necessary to state our theorems. Let $K$ be an alphabet, i.e., a non-empty finite set of symbols. $K^{(n)}$ and $K^{\omega}$ denote the set of all finite words $w$ with length $|w|=n$ and the set of all $\omega$-words $w_{1} w_{2} \ldots w_{i} \ldots\left(w_{i} \in K\right)$. In particular, $K^{(0)}=\{\lambda\}$, where $\lambda$ is the empty word. We put

$$
K^{*}:=\bigcup_{n=0}^{\infty} K^{(n)}, \quad K^{+}:=\bigcup_{n=1}^{\infty} K^{(n)}, \quad K^{\infty}:=K^{*} \cup K^{\omega} .
$$

$K^{*}$ is a free monoid generated by $K$ with the operation of concatenation and with the unit $\lambda . K^{\infty}$ is a complete metric space with metric $d$ defined by

$$
d(u, v):=\left(\inf \left\{n ; u_{n} \neq v_{n}\right\}\right)^{-1}, \quad u \neq v,
$$

for $u=u_{1} u_{2} \ldots u_{i} \ldots, v=v_{1} v_{2} \ldots v_{j} \ldots \in K^{\infty}\left(u_{i}, v_{j} \in K\right)$. 
In what follows, we set $K:=\{a, b, c\}$, and $k, l$ denote fixed integers satisfying (1.1). We define a monoid morphism $\sigma \in \operatorname{Hom}\left(K^{*}, K^{*}\right)$ by

$$
\sigma(a)=a^{k} b, \quad \sigma(b)=a^{l} c, \quad \sigma(c)=a .
$$

$G$ denotes the triple $(K, \sigma, a) . G$ is a DOL system (cf. [13], [14]). It is easily seen that

$$
\sigma^{n+2}(a)=\left(\sigma^{n+1}(a)\right)^{k}\left(\sigma^{n}(a)\right)^{l} \sigma^{n-1}(a), \quad n \geq 1,
$$

where $\sigma^{n}$ denotes the $n$-fold iteration of $\sigma$, so that $\left\{\sigma^{n}(a)\right\}_{n=0}^{\infty}$ is a Cauchy sequence in $K^{\infty}$. We write

$$
w:=\lim _{m \rightarrow \infty} \sigma^{m}(a)=w_{1} w_{2} \ldots w_{n} \ldots \in K^{\omega} \quad\left(w_{n} \in K\right),
$$

which will be referred to as the $\omega$-word generated by $G$. We denote the word $w$ by $w(a, b, c)$ to specify the symbols $a, b, c$. For an integer $g \geq 2$, let $w\left(u_{1}, u_{2}, u_{3}\right)$ stand for the infinite sequence of integers in $\{0,1, \ldots, g-1\}=$ : $L$, say, obtained by replacing the symbols $a, b, c$ appearing in $w(a, b, c)$ with $u_{1}, u_{2}, u_{3} \in L^{+}$, respectively. Define the number $0 . w\left(u_{1}, u_{2}, u_{3}\right)$ by means of the $g$-adic expansion:

$$
0 . w\left(u_{1}, u_{2}, u_{3}\right):=\sum_{n=1}^{\infty} c_{n} g^{-n},
$$

where $w\left(u_{1}, u_{2}, u_{3}\right)=c_{1} c_{2} \ldots c_{n} \ldots \in L^{\omega}\left(c_{n} \in L\right)$.

We define the linear recurrence sequence $\left\{f_{n}\right\}_{n=-2}^{\infty}$ by

$$
\begin{gathered}
f_{n}=k f_{n-1}+l f_{n-2}+f_{n-3}, \quad n \in \mathbb{N}, \\
\left(f_{-2}, f_{-1}, f_{0}\right)=\left(l^{2}-l-k+1,1-l, 1\right) .
\end{gathered}
$$

Then it is easily seen that

$$
\left|\sigma^{n-1}(a)\right|=f_{n}, \quad n \in \mathbb{N} .
$$

We denote by $\mathcal{A}_{n}, \mathcal{B}_{n}, \mathcal{C}_{n}$ (resp. $\mathcal{A}, \mathcal{B}, \mathcal{C}$ ) the characteristic sets of the word $\sigma^{n-1}(a)=w_{1} w_{2} \ldots w_{m} \ldots w_{f_{n}}\left(\right.$ resp. $\left.w=w_{1} w_{2} \ldots w_{m} \ldots \in K^{\omega}\right)$ defined by

$$
\begin{gathered}
\mathcal{A}_{n}:=\left\{m ; w_{m}=a, 1 \leq m \leq f_{n}\right\}, \\
\mathcal{B}_{n}:=\left\{m ; w_{m}=b, 1 \leq m \leq f_{n}\right\}, \\
\mathcal{C}_{n}:=\left\{m ; w_{m}=c, 1 \leq m \leq f_{n}\right\}, \\
\mathcal{A}=\bigcup_{n=1}^{\infty} \mathcal{A}_{n}, \quad \mathcal{B}=\bigcup_{n=1}^{\infty} \mathcal{B}_{n}, \quad \mathcal{C}=\bigcup_{n=1}^{\infty} \mathcal{C}_{n} .
\end{gathered}
$$

It is clear that $\mathcal{A}, \mathcal{B}$ and $\mathcal{C}$ give a partition of $\mathbb{N}$.

In this paper, we consider the algorithm of Jacobi-Perron (resp. of Parusnikov), by means of which a given vector ${ }^{t}(\varphi, \psi) \in \mathbb{R}^{2}\left(\operatorname{resp} .{ }^{t}(\varphi, \psi) \in \mathbb{K}^{2}\right.$, where $\mathbb{K}=\mathbb{C}\left(\left(z^{-1}\right)\right)=$ the field of formal Laurent series) can be developed 
in a higher-dimensional continued fraction

$$
\left[\begin{array}{l}
a_{0} ; a_{1}, a_{2}, \ldots, a_{n}, \ldots \\
b_{0} ; b_{1}, b_{2}, \ldots, b_{n}, \ldots
\end{array}\right]
$$

where $a_{i}, b_{i} \in \mathbb{Z}$ (resp. $a_{i}, b_{i} \in \mathbb{C}[z]$ ). Precise notations and definitions of these algorithms are given in Section 3.

Now, we can state our results:

Theorem 1. Let $g \geq 1$ be an integer and let $a_{n}, b_{n}$ be integers defined by

$$
\begin{array}{ll}
a_{n}=g^{f_{n-3}} \sum_{j=0}^{l-1} g^{f_{n-2} \cdot j}, & n \geq 3, \\
b_{n}=g^{l f_{n-2}+f_{n-3}} \sum_{j=0}^{k-1} g^{f_{n-1} \cdot j}, & n \geq 2,
\end{array}
$$

where $f_{n}$ is the sequence given by (2.3) with the conditions (1.1). Let $\varphi(g)$ and $\psi(g)$ be the numbers defined by the expression in the Jacobi-Perron algorithm

$$
\left(\begin{array}{l}
\varphi(g) \\
\psi(g)
\end{array}\right):=\left[\begin{array}{l}
0 ; 1,1, a_{3}, \ldots, a_{n}, \ldots \\
0 ; 1, b_{2}, b_{3}, \ldots, b_{n}, \ldots
\end{array}\right] .
$$

The we have the following statements:

(i) When $g \geq 2, \varphi(g)$ and $\psi(g)$ are transcendental numbers and their g-adic expansions are given by

$$
\varphi(g)=0 . w(g-1,0,0), \quad \psi(g)=0 . w(g-1, g-1,0) .
$$

Moreover, $\varphi(1)$ and $\psi(1)$ are algebraic numbers of the third degree, where $w$ is the $\omega$-word generated by the DOL system $G=(K, \sigma, a)$.

(ii) $1, \varphi(g)$ and $\psi(g)$ are linearly independent over $\mathbb{Q}$ for each $g \in \mathbb{N}$.

(iii) The expression (2.7) is admissible, i.e., the right-hand side of (2.7) is derived from the Jacobi-Perron algorithm.

(iv) The $n$-th convergent of (2.7) is given by

$$
\left(\sum_{m=1}^{f_{n}} g^{-m}\right)^{-1} \cdot{ }^{t}\left(\sum_{m \in \mathcal{A}_{n}} g^{-m}, \sum_{m \in \mathcal{A}_{n} \cup \mathcal{B}_{n}} g^{-m}\right) .
$$

Theorem 2. Let $\mathcal{A}, \mathcal{B}$, and $\mathcal{C}$ be the characteristic sets of the word $w \in K^{\omega}$ given by (2.5), and let $\eta(z), \xi(z)$ be the functions defined by

$$
\eta(z)=\varphi_{\mathcal{B}}(z) / \varphi_{\mathcal{A}}(z), \quad \xi(z)=\left(\varphi_{\mathcal{B}}(z)+\varphi_{\mathcal{C}}(z)\right) / \varphi_{\mathcal{A}}(z)
$$

where $\varphi_{\mathcal{D}}(z)$ denotes the function $\sum_{m \in \mathcal{D}} z^{-m}$ for a given subset $\mathcal{D}$ of $\mathbb{N}$. Then we have the following statements: 
(i) $\varphi_{\mathcal{A}}(z), \varphi_{\mathcal{B}}(z)$, and $\varphi_{\mathcal{C}}(z)$ are transcendental functions over $\mathbb{C}(z)$ with the unit circle $|z|=1$ as their natural boundary.

(ii) $\varphi_{\mathcal{A}}(z), \varphi_{\mathcal{B}}(z)$, and $\varphi_{\mathcal{C}}(z)$ are linearly independent over $\mathbb{C}(z)$.

(iii) The admissible Jacobi-Perron-Parusnikov expansion for ${ }^{t}(\eta(z)$, $\xi(z))$ is given by

$$
\left(\begin{array}{l}
\eta(z) \\
\xi(z)
\end{array}\right):=\left[\begin{array}{l}
0 ; 1, a_{3}, a_{4}, \ldots, a_{n}, \ldots \\
0 ; b_{2}, b_{3}, b_{4}, \ldots, b_{n}, \ldots
\end{array}\right]
$$

where $a_{n}(n \geq 3)$ and $b_{n}(n \geq 2)$ are polynomials in $z$ obtained by replacing $g$ by $z$ in (2.6).

(iv) The $n$-th convergent of (2.9) is given by

$$
\left(\sum_{m \in \mathcal{A}_{n}} z^{-m}\right)^{-1} \cdot{ }^{t}\left(\sum_{m \in \mathcal{B}_{n}} z^{-m}, \sum_{m \in \mathcal{B}_{n} \cup \mathcal{C}_{n}} z^{-m}\right) .
$$

The transcendence result (i) in Theorem 1 can be generalized:

Theorem 3. Let $g \geq 2$ be an integer and let $L=\{0,1, \ldots, g-1\}$. For any given $u_{1}, u_{2}, u_{3} \in L^{+}, M$ denotes the matrix $\left(\left|u_{i}\right|_{j}\right)_{1 \leq i \leq 3,0 \leq j \leq g-1}$, where $\left|u_{i}\right|_{j}$ indicates the number of $j \in L$ appearing in the word $u_{i}$. Suppose that $\operatorname{rank} M \geq 2$. Then the number defined by the g-adic expansion $0 . w\left(u_{1}, u_{2}, u_{3}\right)$ is transcendental.

3. Jacobi-Perron algorithms. In this section, we define two kinds of continued fraction expansions of higher dimension. We use the following notations.

$\mathbb{K}=\mathbb{C}\left(\left(z^{-1}\right)\right)$, the field of formal Laurent series with complex coefficients. $\mathbb{K}$ is a metric space with the distance function $d_{\mathbb{K}}(\varphi, \psi):=\|\varphi-\psi\|$ $(\varphi, \psi \in \mathbb{K})$, where \|\| is the usual non-archimedean norm defined by $\|\varphi\|:=$ $e^{-k}$ for $0 \neq \varphi=\sum_{m=k}^{\infty} c_{m} z^{-m} \in \mathbb{K}$ with $c_{k} \neq 0, k \in \mathbb{Z}$ and $\|0\|:=0$.

$[\varphi]:=$ the polynomial part of $\varphi \in \mathbb{K}$, i.e., $[\varphi]:=0$ for $\|\varphi\|<1$ and $[\varphi]:=\sum_{m=k}^{0} c_{m} z^{-m}$ for $\|\varphi\| \geq 1$ with $\varphi=\sum_{m=k}^{\infty} c_{m} z^{-m}$.

$\langle\varphi\rangle:=\varphi-[\varphi]$, the fractional part of $\varphi \in \mathbb{K}$. We denote by $[\varphi]$ and $\langle\varphi\rangle$ the vectors $^{t}([\varphi],[\psi])$ and ${ }^{t}(\langle\varphi\rangle,\langle\psi\rangle)$, respectively for $\varphi={ }^{t}(\varphi, \psi) \in \mathbb{K}^{2}$, where ${ }^{t}(\varphi, \psi)$ is the transposed vector of $(\varphi, \psi)$. $T$ denotes the transformation defined by

$$
T\left({ }^{t}(\varphi, \psi)\right):={ }^{t}(1 / \psi, \varphi / \psi) \quad(0 \neq \psi, \varphi \in \mathbb{K}) .
$$

Furthermore, we denote $T\left({ }^{t}(\varphi, \psi)\right)$ by $1 /{ }^{t}(\varphi, \psi)$.

Following Parusnikov [12, 9], we define the expansion of $\varphi={ }^{t}(\varphi, \psi) \in \mathbb{K}^{2}$ in the Jacobi-Perron algorithm. We put $\varphi_{0}={ }^{t}\left(\varphi_{0}, \psi_{0}\right):={ }^{t}(\langle\varphi\rangle,\langle\psi\rangle)$ and

$$
\varphi_{n} \equiv{ }^{t}\left(\varphi_{n}, \psi_{n}\right):=S^{n}\left(\varphi_{0}\right)
$$


if $\varphi_{n} \neq 0$ for all $n \geq 0$, where $S^{n}$ denotes the $n$-fold iteration of $S$, which is defined by $S\left(\boldsymbol{\varphi}_{0}\right):=\left\langle T^{-1}\left(\boldsymbol{\varphi}_{0}\right)\right\rangle\left(S^{0}=\right.$ the identity map on $\left.\mathbb{K}\right)$. Then we define $\boldsymbol{a}_{n} \equiv{ }^{t}\left(a_{n}, b_{n}\right) \in(\mathbb{C}[z])^{2}$ by

$$
\boldsymbol{a}_{0}:=[\boldsymbol{\varphi}], \quad \boldsymbol{a}_{n}:=\left[T^{-1}\left(S^{n-1}\left(\boldsymbol{\varphi}_{0}\right)\right)\right] \quad(n \geq 1) .
$$

The vector $\pi_{n} \in(\mathbb{C}(z))^{2}$ defined by

$$
\begin{aligned}
& \pi_{n}=\pi_{n}(\boldsymbol{\varphi})=\boldsymbol{a}_{0}+\frac{1}{\boldsymbol{a}_{1}+\frac{1}{\boldsymbol{a}_{2}+}} \\
& +\frac{1}{\boldsymbol{a}_{n}}
\end{aligned}
$$

converges componentwise to $\varphi$ as $n \rightarrow \infty$ by Parusnikov's theorem in [12], where the convergence is with respect to the metric $d_{\mathbb{K}}$. Thus, we may write

$$
\boldsymbol{\varphi}=\boldsymbol{a}_{0}+\frac{1}{\boldsymbol{a}_{1}+\frac{1}{\boldsymbol{a}_{2}+\quad}}
$$

(3.2) and (3.3) will be also written as

$$
\begin{gathered}
\pi_{n}\left(\begin{array}{l}
\varphi \\
\psi
\end{array}\right)=\left[\begin{array}{l}
a_{0} ; a_{1}, a_{2}, \ldots, a_{n} \\
b_{0} ; b_{1}, b_{2}, \ldots, b_{n}
\end{array}\right], \\
\left(\begin{array}{c}
\varphi \\
\psi
\end{array}\right):=\left[\begin{array}{l}
a_{0} ; a_{1}, a_{2}, \ldots, a_{n}, \ldots \\
b_{0} ; b_{1}, b_{2}, \ldots, b_{n}, \ldots
\end{array}\right] .
\end{gathered}
$$

The right-hand side of (3.3) will be called the expression of $\varphi \in \mathbb{K}^{2}$ in the Jacobi-Perron-Parusnikov algorithm (abbr. JPP algorithm). Apart from the algorithm given by (3.1), we can consider the expression (3.3) for any given sequence of vectors $\boldsymbol{a}_{1}, \boldsymbol{a}_{2}, \ldots$ in $\mathbb{K}^{2}$ provided that its $n$th convergent (3.2) is well-defined and converges to some element of $\mathbb{K}^{2}$. The JPP expression (3.3) will be called admissible if it is derived from the algorithm (3.1).

Denote by $A_{n}$ and $P_{n}$ the $3 \times 3$ matrices given by

$$
\begin{gathered}
A_{n}:=\left(\begin{array}{ccc}
0 & 0 & 1 \\
1 & 0 & a_{n} \\
0 & 1 & b_{n}
\end{array}\right) \in \mathrm{SL}(3, \mathbb{C}[z]), \\
P_{n}:=A_{1} A_{2} \ldots A_{n}, \quad n \geq 1,
\end{gathered}
$$


and $P_{0}:=E$, the $3 \times 3$ unit matrix. Set

$$
P_{n}:=\left(\begin{array}{lll}
p_{n-2} & p_{n-1} & p_{n} \\
q_{n-2} & q_{n-1} & q_{n} \\
r_{n-2} & r_{n-1} & r_{n}
\end{array}\right) \in \operatorname{SL}(3, \mathbb{C}[z]) .
$$

Then we have

$$
\pi_{n}\left(\begin{array}{l}
\varphi \\
\psi
\end{array}\right)=\left(\begin{array}{c}
a_{0} \\
b_{0}
\end{array}\right)+\left(\begin{array}{c}
p_{n} / r_{n} \\
q_{n} / r_{n}
\end{array}\right) \in(\mathbb{C}(z))^{2}
$$

where $\left\{p_{n}\right\}_{n=-2}^{\infty},\left\{q_{n}\right\}_{n=-2}^{\infty},\left\{r_{n}\right\}_{n=-2}^{\infty}$ are the sequences of polynomials satisfying the recurrence relations

$$
p_{n}=b_{n} p_{n-1}+a_{n} p_{n-2}+p_{n-3}, \quad n \geq 1,
$$

and the same recurrences with $q_{n}$ and $r_{n}$ in place of $p_{n}$ with the initial condition $P_{0}=E$.

If we take the field $\mathbb{R}$ of real numbers for $\mathbb{K}$ and define $[\varphi]$ to be the integral part of $\varphi \in \mathbb{R}$, we have an algorithm, which is the simplest JacobiPerron algorithm (abbr. JP algorithm).

\section{Pisot numbers of third degree}

LEMMA 4.1. Let $p(x)=x^{3}-k x^{2}-l x-1$ be a polynomial satisfying the conditions (1.1). Then $p(x)$ is irreducible over $\mathbb{Q}$ and has a real root $\alpha$ such that $k<\alpha<k+1$ and $|\beta|,|\gamma|<1$, where $\beta, \gamma$ are the algebraic conjugates of $\alpha$, i.e., $\alpha$ is a Pisot number.

Proof. Since $p(k) \leq-1$ and $p(k+1) \geq k, p(x)$ has a real root $\alpha$ with $k<\alpha<k+1$. Put $\xi:=\left(k-\sqrt{k^{2}+3 l}\right) / 3$, so that $(d / d x) p(\xi)=0$ and $-2 / 3<\xi \leq 0$. We have $-1<\beta \leq \gamma<0$ if $p(\xi) \geq 0$, otherwise $\beta, \gamma \notin \mathbb{R}$, $|\beta|=|\gamma|=1 / \sqrt{\alpha}<1$, since $p(-1)=-k+l-2<0$ and $p(0)=-1<0$. In any case $\alpha, \beta, \gamma \notin \mathbb{Z}$. Thus, $p(x)$ is irreducible.

Remark. It can be proved that the inequalities $k \geq l>\sqrt{4 k+3}$ $(k \geq 5)$ imply $\beta, \gamma \in \mathbb{R}$, and $\sqrt{3 k} \geq l(k \geq 3)$ implies $\beta, \gamma \notin \mathbb{R}$.

Lemma 4.2. Let $\lambda=1 / \alpha$ and $k=l / \alpha+1 / \alpha^{2}$, where $\alpha>1$ is the root of $p(x)$ given in Lemma 4.1. Then the following expression is admissible in the JP algorithm:

$$
\left(\begin{array}{l}
\lambda \\
\kappa
\end{array}\right):=\left[\begin{array}{llll}
0 ; & l, l & l, & l,
\end{array}\right] .
$$

Furthermore, writing $\pi_{n}{ }^{t}(\lambda, \kappa)={ }^{t}\left(p_{n} / r_{n}, q_{n} / r_{n}\right)$ as in Section 3 , we have

$$
\lambda-p_{n} / r_{n}=O\left(r_{n}^{-1-\delta}\right), \quad \kappa-q_{n} / r_{n}=O\left(r_{n}^{-1-\delta}\right)
$$

where $\delta:=-\log |\beta| / \log \alpha(>0)$, and $\beta, \gamma$ are the algebraic conjugates of $\alpha$ with $|\beta| \geq|\gamma|$. 
Proof. We have

$$
T^{-1}\left({ }^{t}(\lambda, \kappa)\right)={ }^{t}(l+1 / \alpha, \alpha)={ }^{t}(l, k)+{ }^{t}(\lambda, \kappa),
$$

with $0<\lambda<1$ and $0<\kappa<1$, which leads to the admissible expression (4.1). Noting that the characteristic polynomial of the recurrence sequences $p_{n}, q_{n}, r_{n}$ is $p(x)$, we can write

$$
\begin{aligned}
& p_{n}=\mu \alpha^{n-1}+O\left(|\beta|^{n}\right), \\
& q_{n}=\nu \alpha^{n}+O\left(|\beta|^{n}\right), \\
& r_{n}=p_{n+1}=\mu \alpha^{n}+O\left(|\beta|^{n}\right)
\end{aligned}
$$

with positive numbers $\mu, \nu$. Since

$$
\lambda r_{n}-p_{n}=\frac{\kappa\left(p_{n-1} r_{n}-p_{n} r_{n-1}\right)+\lambda\left(p_{n-2} r_{n}-p_{n} r_{n-2}\right)}{r_{n}+\kappa r_{n-1}+\lambda r_{n-2}},
$$

we have

$$
\lambda r_{n}-p_{n}=O\left(|\beta|^{n}\right) .
$$

Similarly we get

$$
\kappa r_{n}-p_{n}=O\left(|\beta|^{n}\right) .
$$

(4.2) follows from these inequalities.

5. DOL system $G$ and related polynomials. We define the polynomials $\widetilde{p}_{n}=\widetilde{p}_{n}(z), \widetilde{q}_{n}=\widetilde{q}_{n}(z), \widetilde{r}_{n}=\widetilde{r}_{n}(z)$ by

$$
\widetilde{p}_{n}:=\sum_{m \in \mathcal{A}_{n}} z^{m}, \quad \widetilde{q}_{n}:=\sum_{m \in \mathcal{B}_{n}} z^{m}, \quad \widetilde{r}_{n}:=\sum_{m \in \mathcal{C}_{n}} z^{m},
$$

where $\mathcal{A}_{n}, \mathcal{B}_{n}, \mathcal{C}_{n}$ are the characteristic sets of $\sigma^{n-1}(a)$ defined by (2.5). For brevity, we put

$$
(h)_{m}:=\sum_{i=0}^{m-1} h^{i}, \quad(h)_{0}:=0 \quad(h \in \mathbb{C}(z)) .
$$

Then, from (2.2) and (2.4), we have

LEMMA 5.1.

$$
\widetilde{p}_{n}=\widetilde{c}_{n} \widetilde{p}_{n-1}+\widetilde{b}_{n} \widetilde{p}_{n-2}+\widetilde{a}_{n} \widetilde{p}_{n-3}, \quad n \geq 4,
$$

and the same recurrences hold with $\widetilde{q}_{n}$ and $\widetilde{r}_{n}$ in place of $\widetilde{p}_{n}$ with the initial conditions

$$
\left(\begin{array}{ccc}
\widetilde{p}_{1} & \widetilde{p}_{2} & \widetilde{p}_{3} \\
\widetilde{q}_{1} & \widetilde{q}_{2} & \widetilde{q}_{3} \\
\widetilde{r}_{1} & \widetilde{r}_{2} & \widetilde{r}_{3}
\end{array}\right)=\left(\begin{array}{ccc}
z & z \cdot(z)_{k} & z \cdot(z)_{k} \cdot\left(z^{k+1}\right)_{k}+z^{k(k+1)+1} \cdot(z)_{l} \\
0 & z^{k+1} & z^{k+1} \cdot\left(z^{k+1}\right)_{k} \\
0 & 0 & z^{k^{2}+k+l+1}
\end{array}\right)
$$


where

$$
\begin{aligned}
& \widetilde{a}_{n}=z^{k f_{n-1}+l f_{n-2}} \quad\left(=z^{f_{n}-f_{n-3}}\right), \\
& \widetilde{b}_{n}=z^{k f_{n-1}} \cdot\left(z^{f_{n-2}}\right)_{l}, \\
& \widetilde{c}_{n}=\left(z^{f_{n-1}}\right)_{k},
\end{aligned}
$$

and $f_{n}$ is the sequence defined by (2.3).

We put

$$
\begin{aligned}
& \bar{p}_{n}(z)=z^{f_{n}} \widetilde{p}_{n}\left(z^{-1}\right), \\
& \bar{q}_{n}(z)=z^{f_{n}} \widetilde{q}_{n}\left(z^{-1}\right), \\
& \bar{r}_{n}(z)=z^{f_{n}} \widetilde{r}_{n}\left(z^{-1}\right) .
\end{aligned}
$$

Then $\bar{p}_{n}, \bar{q}_{n}$, and $\bar{r}_{n}$ are polynomials in $z$. It follows from (5.2) that

$$
\begin{aligned}
\bar{p}_{n}(z)= & z^{f_{n}-f_{n-3}} \widetilde{a}_{n}\left(z^{-1}\right) \bar{p}_{n-3}(z)+z^{f_{n}-f_{n-2}} \widetilde{b}_{n}\left(z^{-1}\right) \bar{p}_{n-2}(z) \\
& +z^{f_{n}-f_{n-1}} \widetilde{c}_{n}\left(z^{-1}\right) \bar{p}_{n-1}(z),
\end{aligned}
$$

which together with (5.4) yields the recurrence relations

$$
\bar{p}_{n}=b_{n} \bar{p}_{n-1}+a_{n} \bar{p}_{n-2}+\bar{p}_{n-3}, \quad n \geq 4,
$$

and the same recurrences with $\bar{q}_{n}$ and $\bar{r}_{n}$ in place of $\bar{p}_{n}$, where

$$
a_{n}=z^{f_{n-3}} \cdot\left(z^{f_{n-2}}\right)_{l}, \quad b_{n}=z^{l f_{n-2}+f_{n-3}} \cdot\left(z^{f_{n-1}}\right)_{k} .
$$

In view of (5.3), we have the initial conditions

$$
\left(\begin{array}{ccc}
\bar{p}_{1} & \bar{p}_{2} & \bar{p}_{3} \\
\bar{q}_{1} & \bar{q}_{2} & \bar{q}_{3} \\
\bar{r}_{1} & \bar{r}_{2} & \bar{r}_{3}
\end{array}\right)=\left(\begin{array}{ccc}
1 & z \cdot(z)_{k} & z \cdot(z)_{k} \cdot z^{l+1}\left(z^{k+1}\right)_{k}+z \cdot(z)_{l} \\
0 & 1 & z^{l+1} \cdot\left(z^{k+1}\right)_{k} \\
0 & 0 & 1
\end{array}\right)
$$

Now, we set

$$
\begin{aligned}
\bar{P}_{n}:= & \left(\begin{array}{lll}
\bar{p}_{n-2} & \bar{p}_{n-1} & \bar{p}_{n} \\
\bar{q}_{n-2} & \bar{q}_{n-1} & \bar{q}_{n} \\
\bar{r}_{n-2} & \bar{r}_{n-1} & \bar{r}_{n}
\end{array}\right), \quad \Delta:=\left(\begin{array}{ccc}
1 & 0 & 0 \\
a_{1} & 1 & 0 \\
b_{1} & a_{2} & 1
\end{array}\right), \\
& \bar{P}_{0}:=\Delta^{-1}=\left(\begin{array}{ccc}
1 & 0 & 0 \\
-a_{1} & 1 & 0 \\
a_{1} a_{2}-b_{1} & -a_{2} & 1
\end{array}\right) .
\end{aligned}
$$

Then the relations (5.6) for $\bar{p}_{n}, \bar{q}_{n}$ and $\bar{r}_{n}$ hold for all $n \geq 1$ by (5.7), (5.8) and (2.3).

Therefore, we get

$$
\bar{P}_{n}=\Delta^{-1} A_{1} A_{2} \ldots A_{n} \quad(n \in \mathbb{N}), \quad \bar{P}_{0}=\Delta^{-1},
$$


where

$$
A_{n}:=\left(\begin{array}{ccc}
0 & 0 & 1 \\
1 & 0 & a_{n} \\
0 & 1 & b_{n}
\end{array}\right) .
$$

We define the sequence of rational functions $p_{n}=p_{n}(z), q_{n}=q_{n}(z)$ and $r_{n}=r_{n}(z)$ by

$$
P_{n}:=\Delta \bar{P}_{n}, \quad P_{n}=:\left(\begin{array}{ccc}
p_{n-2} & p_{n-1} & p_{n} \\
q_{n-2} & q_{n-1} & q_{n} \\
r_{n-2} & r_{n-1} & r_{n}
\end{array}\right), \quad n \geq 0 .
$$

By the definitions (5.9) and (5.10), we have

$$
P_{n}=A_{1} A_{2} \ldots A_{n} \quad(n \in \mathbb{N}), \quad P_{0}=E,
$$

where $E$ is the $3 \times 3$ unit matrix. Therefore, ${ }^{t}\left(p_{n}(z) / r_{n}(z), q_{n}(z) / r_{n}(z)\right)$ is the $n$th convergent of the JPP expression

$$
\left(\begin{array}{c}
\widetilde{\varphi}(z) \\
\widetilde{\psi}(z)
\end{array}\right)=\left[\begin{array}{l}
0 ; a_{1}, a_{2}, \ldots, a_{n}, \ldots \\
0 ; b_{1}, b_{2}, \ldots, b_{n}, \ldots
\end{array}\right]
$$

(see (3.4), (3.5)). It follows from (5.10), (5.5) and (5.1) that

$$
\begin{aligned}
& p_{n}(z) / r_{n}(z)=\widetilde{p}_{n}\left(z^{-1}\right) /\left(b_{1}(z) \widetilde{p}_{n}\left(z^{-1}\right)+a_{2}(z) \widetilde{q}_{n}\left(z^{-1}\right)+\widetilde{r}_{n}\left(z^{-1}\right)\right), \\
& q_{n}(z) / r_{n}(z) \\
& \quad=\left(a_{1}(z) \widetilde{p}_{n}\left(z^{-1}\right)+\widetilde{q}_{n}\left(z^{-1}\right)\right) /\left(b_{1}(z) \widetilde{p}_{n}\left(z^{-1}\right)+a_{2}(z) \widetilde{q}_{n}\left(z^{-1}\right)+\widetilde{r}_{n}\left(z^{-1}\right)\right),
\end{aligned}
$$

and

$$
\begin{aligned}
\lim _{n \rightarrow \infty} \widetilde{p}_{n}\left(z^{-1}\right)= & \sum_{m \in \mathcal{A}} z^{-m}, \quad \lim _{n \rightarrow \infty} \widetilde{q}_{n}\left(z^{-1}\right)=\sum_{m \in \mathcal{B}} z^{-m}, \\
& \lim _{n \rightarrow \infty} \widetilde{r}_{n}\left(z^{-1}\right)=\sum_{m \in \mathcal{C}} z^{-m} .
\end{aligned}
$$

Since the above series converge in the region $|z|>1$, the right-hand side expression in (5.11) converges in $|z|>1$, and hence $\widetilde{\varphi}(z)$ and $\widetilde{\psi}(z)$ are holomorphic functions on $|z|>1$ given by

$$
\begin{aligned}
\widetilde{\varphi}(z) & =\frac{\sum_{m \in \mathcal{A}} z^{-m}}{b_{1}(z) \sum_{m \in \mathcal{A}} z^{-m}+a_{2}(z) \sum_{m \in \mathcal{B}} z^{-m}+\sum_{m \in \mathcal{C}} z^{-m}}, \\
\widetilde{\psi}(z) & =\frac{a_{1}(z) \sum_{m \in \mathcal{A}} z^{-m}+\sum_{m \in \mathcal{B}} z^{-m}}{b_{1}(z) \sum_{m \in \mathcal{A}} z^{-m}+a_{2}(z) \sum_{m \in \mathcal{B}} z^{-m}+\sum_{m \in \mathcal{C}} z^{-m}} .
\end{aligned}
$$

We note that the partial denominators $a_{n}(n \geq 3), b_{n}(n \geq 2)$ in the JPP expression (5.11) are polynomials in $z$. But $a_{1}, a_{2}, b_{1}$ may not be 
polynomials for certain $k, l$. However, it follows from (5.12) that

$$
\begin{aligned}
& T^{-1}\left(\begin{array}{l}
p_{n}(z) / r_{n}(z) \\
q_{n}(z) / r_{n}(z)
\end{array}\right) \\
& =\left(\begin{array}{l}
a_{1}(z) \\
b_{1}(z)
\end{array}\right)+\left(\begin{array}{c}
\widetilde{q}_{n}\left(z^{-1}\right) / \widetilde{p}_{n}\left(z^{-1}\right) \\
\left(a_{2}(z) \widetilde{q}_{n}\left(z^{-1}\right)+\widetilde{r}_{n}\left(z^{-1}\right)\right) / \widetilde{p}_{n}\left(z^{-1}\right)
\end{array}\right), \\
& T^{-1}\left(T^{-1}\left(\begin{array}{c}
p_{n}(z) / r_{n}(z) \\
q_{n}(z) / r_{n}(z)
\end{array}\right)-\left(\begin{array}{c}
a_{1}(z) \\
b_{1}(z)
\end{array}\right)\right) \\
& =\left(\begin{array}{c}
a_{2}(z) \\
0
\end{array}\right)+\left(\begin{array}{l}
\widetilde{r}_{n}\left(z^{-1}\right) / \widetilde{q}_{n}\left(z^{-1}\right) \\
\widetilde{p}_{n}\left(z^{-1}\right) / \widetilde{q}_{n}\left(z^{-1}\right)
\end{array}\right),
\end{aligned}
$$

$n \geq 3$. Thus, we have

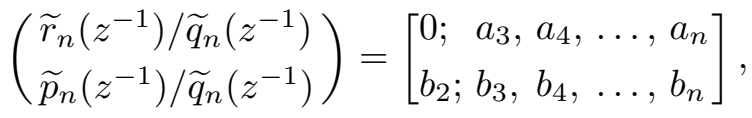

since ${ }^{t}\left(p_{n}(z) / r_{n}(z), q_{n}(z) / r_{n}(z)\right)$ is the $n$th convergent of (5.11). On the other hand,

$$
\begin{aligned}
T^{-1}\left(\begin{array}{c}
\varphi_{n}(z) \\
\psi_{n}(z)
\end{array}\right) & =\left(\begin{array}{c}
\sum_{m \in \mathcal{A}_{n} \cup \mathcal{B}_{n}} z^{-m} / \sum_{m \in \mathcal{A}_{n}} z^{-m} \\
\sum_{m \in \mathcal{A}_{n} \cup \mathcal{B}_{n} \cup \mathcal{C}_{n}} z^{-m} / \sum_{m \in \mathcal{A}_{n}} z^{-m}
\end{array}\right) \\
& =\left(\begin{array}{c}
1 \\
1
\end{array}\right)+\left(\begin{array}{c}
\sum_{m \in \mathcal{B}_{n}} z^{-m} / \sum_{m \in \mathcal{A}_{n}} z^{-m} \\
\sum_{m \in \mathcal{B}_{n} \cup \mathcal{C}_{n}} z^{-m} / \sum_{m \in \mathcal{A}_{n}} z^{-m}
\end{array}\right), \quad n \geq 1,
\end{aligned}
$$

where $\varphi_{n}(z)$ and $\psi_{n}(z)$ are the rational functions given by

$$
\begin{aligned}
& \varphi_{n}(z):=\sum_{m \in \mathcal{A}_{n}} z^{-m} / \sum_{m \in \mathcal{A}_{n} \cup \mathcal{B}_{n} \cup \mathcal{C}_{n}} z^{-m}, \\
& \psi_{n}(z):=\sum_{m \in \mathcal{A}_{n} \cup \mathcal{B}_{n}} z^{-m} / \sum_{m \in \mathcal{A}_{n} \cup \mathcal{B}_{n} \cup \mathcal{C}_{n}} z^{-m} .
\end{aligned}
$$

Hence we have

$$
\begin{aligned}
T^{-1}\left(T^{-1}\left(\begin{array}{l}
\varphi_{n}(z) \\
\psi_{n}(z)
\end{array}\right)-\right. & \left.\left(\begin{array}{l}
1 \\
1
\end{array}\right)\right) \\
& =\left(\begin{array}{l}
1 \\
0
\end{array}\right)+\left(\begin{array}{l}
\sum_{m \in \mathcal{C}_{n}} z^{-m} / \sum_{m \in \mathcal{B}_{n}} z^{-m} \\
\sum_{m \in \mathcal{A}_{n}} z^{-m} / \sum_{m \in \mathcal{B}_{n}} z^{-m}
\end{array}\right),
\end{aligned}
$$

$n \geq 2$. By (5.15), (5.1) and (5.13), we get

$$
T^{-1}\left(T^{-1}\left(\begin{array}{l}
\varphi_{n}(z) \\
\psi_{n}(z)
\end{array}\right)-\left(\begin{array}{l}
1 \\
1
\end{array}\right)\right)=\left[\begin{array}{ll}
1 ; & a_{3}, \ldots, a_{n} \\
b_{2} ; & b_{3}, \ldots, b_{n}
\end{array}\right], \quad n \geq 3,
$$

that is

$$
\left(\begin{array}{l}
\varphi_{n}(z) \\
\psi_{n}(z)
\end{array}\right)=\left[\begin{array}{l}
0 ; 1,1, a_{3}, \ldots, a_{n} \\
0 ; 1, b_{2}, b_{3}, \ldots, b_{n}
\end{array}\right],
$$

which holds for all $n \geq 1$. 


\section{Proof of the theorems}

Proof of Theorem 1 . We define the functions $\varphi(z)$ and $\psi(z)$ by

$$
\left(\begin{array}{l}
\varphi(z) \\
\psi(z)
\end{array}\right)=\left[\begin{array}{l}
0 ; 1,1, a_{3}, \ldots, a_{n}, \ldots \\
0 ; 1, b_{2}, b_{3}, \ldots, b_{n}, \ldots
\end{array}\right]
$$

where $a_{n}(n \geq 3)$ and $b_{n}(n \geq 2)$ are the polynomials in $z$ given by (5.7). Since (5.16) is the $n$th convergent of (6.1), we have by (5.14)

$$
\begin{aligned}
& \lim _{n \rightarrow \infty} \varphi_{n}(z)=(z-1) \cdot \sum_{m \in \mathcal{A}} z^{-m}=\varphi(z), \\
& \lim _{n \rightarrow \infty} \psi_{n}(z)=(z-1) \cdot \sum_{m \in \mathcal{A} \cup \mathcal{B}} z^{-m}=\psi(z),
\end{aligned}
$$

which are holomorphic functions on $|z|>1$. Taking $z=g(2 \leq g \in \mathbb{Z})$ in (6.2), we get (2.8). Furthermore, it follows from (5.16), (5.7), (6.1) and Lemma 4.2 that

$$
\left(\begin{array}{l}
\varphi(1) \\
\psi(1)
\end{array}\right)=\left[\begin{array}{l}
0 ; 1,1, l, l, l, \ldots \\
0 ; 1, k, k, k, k, \ldots
\end{array}\right]=\left[\begin{array}{l}
0 ; 1,1+\lambda \\
0 ; 1,1+\kappa
\end{array}\right],
$$

where $\lambda$ and $\kappa$ are the numbers given in Lemma 4.2. Hence, we have by simple calculation

$$
\varphi(1)=\alpha^{2} /\left(\alpha^{2}+\alpha+1\right), \quad \psi(1)=\left(\alpha^{2}+\alpha\right) /\left(\alpha^{2}+\alpha+1\right),
$$

which are irrationals of third degree, where $\alpha$ is the number given in Lemma 4.1. Thus we obtain the statement (i) of Theorem 1 except for the transcendence. (iv) is clear from (5.14) and (5.16). For the rest of the proof we prepare some lemmas. In view of (5.14) and (6.4), we get the following

LEmma 6.1. Let $\mathcal{A}_{n}, \mathcal{B}_{n}, \mathcal{C}_{n}$ be the characteristic sets (2.5) of the word $\sigma^{n-1}(a)$ and let $\alpha$ be the root of $p(x)$ as in Lemma 4.1. Then

$$
\begin{gathered}
\lim _{n \rightarrow \infty}\left|\mathcal{A}_{n}\right| / f_{n}=\alpha^{2} /\left(\alpha^{2}+\alpha+1\right), \\
\lim _{n \rightarrow \infty}\left|\mathcal{B}_{n}\right| / f_{n}=\alpha /\left(\alpha^{2}+\alpha+1\right), \\
\lim _{n \rightarrow \infty}\left|\mathcal{C}_{n}\right| / f_{n}=1 /\left(\alpha^{2}+\alpha+1\right),
\end{gathered}
$$

where $|\mathcal{D}|$ denotes the number of elements of a finite set $\mathcal{D}$. These numbers are irrationals of third degree.

We define the degree $\varrho_{G}$ of self-similarity of the DOL system $G=$ $(K, \sigma, a)$ by

$$
\varrho_{G}:=\limsup _{n \rightarrow \infty} \frac{d\left(\lim _{m \rightarrow \infty} \sigma^{m}(a), \sigma^{n}(a)^{*}\right)^{-1}}{\left|\sigma^{n}(a)\right|}
$$


where $\sigma^{n}(a)^{*}$ stands for the infinite periodic word $\sigma^{n}(a) \sigma^{n}(a) \ldots \in K^{\omega}$ and $d$ is the distance function of the metric space $K^{\infty}$ described in Section 2 . In the following, we shall show that the quantity $\varrho_{G}$ is connected with the irrationality measure of rational approximation for the numbers $\varphi(g)$ and $\psi(g)$ of Theorem 1. Here, we say that $\mu$ is an irrationality measure of a number $\theta \in \mathbb{R}$ when $|\theta-p / q|<q^{-\mu+\varepsilon}$ for infinitely many coprime integers $p$ and $q$ for any fixed $\varepsilon>0$.

Lemma 6.2. Let $G=(K, \sigma, a)$ be the DOL system given in Section 2 and let $\alpha$ be the number given by Lemma 4.1. Then

$$
\varrho_{G} \geq \begin{cases}k+1 / \alpha & (k \geq 2, l \geq 1) \\ k+1 / \alpha^{2} & (k \geq 2, l=0) \\ 2+1 / \alpha^{3} & (k=l=1)\end{cases}
$$

Proof. We put $u_{n}=\sigma^{n-1}(a)$ for brevity. Then it follows from (2.2) that

$$
w:=\lim _{m \rightarrow \infty} \sigma^{m}(a)=u_{n+1} \ldots=u_{n}^{k} u_{n-1}^{l} u_{n-2} \ldots, \quad n \geq 3 .
$$

Since $u_{n-1}$ and $u_{n-2}$ are prefixes of $u_{n}$, we have

$$
\varrho_{G} \geq \begin{cases}k+f_{n-1} / f_{n} & (l>0), \\ k+f_{n-2} / f_{n} & (l=0) .\end{cases}
$$

We get this lemma for $k \geq 2$ by taking $n \rightarrow \infty$. We write $u \vDash v\left(u, v \in K^{\infty}\right)$ when $v$ is a prefix of $u$. If $k=l=1$, we obtain

$$
\begin{aligned}
w & \vDash u_{n+2}=u_{n+1} u_{n} u_{n-1} \\
& \vDash u_{n} u_{n-1} u_{n-2} u_{n-2} u_{n-3} \\
& =u_{n} u_{n-1} u_{n-2} u_{n-3} u_{n-4} u_{n-5} u_{n-3} \\
& \vDash u_{n} u_{n} u_{n-4} u_{n-5} u_{n-6}=u_{n} u_{n} u_{n-3}
\end{aligned}
$$

for $n \geq 7$, which leads to $\varrho_{G} \geq 2+1 / \alpha^{3}$.

Remark. By a more precise argument, we obtain

$$
w=u_{n} u_{n} u_{n-3} u_{n-6} u_{n-9} \ldots u_{n-3 k},
$$

$n \geq 3 k+4$ when $k=l=1$. Hence, we can prove $\varrho_{G} \geq 2+1 /\left(\alpha^{3}-1\right)=$ $2.1914 \ldots$ when $k=l=1$.

LEMMA 6.3. The irrationality measures of the numbers $\varphi(g)$ and $\psi(g)$ defined by (2.7) with $2 \leq g \in \mathbb{Z}$ are greater than 2 .

Proof. From (5.14), we have the following $g$-adic expansions of $\varphi(g)$ 
and $\psi(g)$ :

$$
\begin{aligned}
\varphi_{n}(g) & =\left((g-1) g^{f_{n}} \sum_{m \in \mathcal{A}_{n}} g^{-m}\right) /\left(g^{f_{n}}-1\right) \\
& =0 . u_{n}^{*}(g-1,0,0), \\
\psi_{n}(g) & =\left((g-1) g^{f_{n}} \sum_{m \in \mathcal{A}_{n} \cup \mathcal{B}_{n}} g^{-m}\right) /\left(g^{f_{n}}-1\right) \\
& =0 . u_{n}^{*}(g-1, g-1,0),
\end{aligned}
$$

where $u_{n}^{*}=u_{n}^{*}(a, b, c)$ is the infinite periodic word $\sigma^{n-1}(a) \sigma^{n-1}(a) \ldots \in K^{\omega}$. Hence, we deduce from (6.2) that

$$
\begin{aligned}
& \left|\varphi(g)-\varphi_{n}(g)\right|<g^{-\left(\varrho_{G}-\varepsilon\right) f_{n}}, \\
& \left|\psi(g)-\psi_{n}(g)\right|<g^{-\left(\varrho_{G}-\varepsilon\right) f_{n}},
\end{aligned}
$$

for all sufficiently large $n$, for any fixed $\varepsilon>0$. Let $s_{n}(g)>0$ and $t_{n}(g)>0$ be the denominators of $\varphi_{n}(g)$ and $\psi_{n}(g)$, respectively, in the expressions of their irreducible fractions. Then it follows from (6.5) that $s_{n}(g)$ and $t_{n}(g)$ are divisors of $g^{f_{n}}-1$. Therefore,

$$
\left|\varphi(g)-\varphi_{n}(g)\right|<s_{n}(g)^{-\varrho_{G}+\varepsilon}, \quad\left|\psi(g)-\psi_{n}(g)\right|<t_{n}(g)^{-\varrho_{G}+\varepsilon},
$$

for infinitely many $n$, for any fixed $\varepsilon>0$. Both $\left\{\varphi_{n}(g)\right\}_{n=1}^{\infty}$ and $\left\{\psi_{n}(g)\right\}_{n=1}^{\infty}$ are infinite sets since $\varphi(g), \psi(g) \notin \mathbb{Q}$, which follows from Lemma 6.1. In view of Lemma 6.2, the proof of the lemma is complete.

Lemma 6.3 together with Roth's theorem implies the transcendence of $\varphi(g)$ and $\psi(g)$; this completes the proof of (i) of Theorem 1.

Next we prove the statement (ii) of Theorem 1. It follows from Lemma 4.2, (6.3) and (6.6) that, for any fixed $g \in \mathbb{N}$,

$$
r_{n}(g) \varphi(g)-p_{n}(g)=o(1), \quad r_{n}(g) \psi(g)-q_{n}(g)=o(1),
$$

where $p_{n}, q_{n}, r_{n}$ are integers given by (5.10). Suppose that $1, \varphi(g)$ and $\psi(g)$ are linearly dependent over $\mathbb{Q}$. Then

$$
x\left(p_{n} / r_{n}+o(1) / r_{n}\right)+y\left(q_{n} / r_{n}+o(1) / r_{n}\right)+z=0,
$$

and hence $x p_{n}+y q_{n}+z r_{n}=0$ for all sufficiently large $n$, for some fixed integers $x, y, z$ with $(x, y, z) \neq(0,0,0)$. This contradicts the fact that $0 \neq \operatorname{det} P_{n}(=1)$.

It remains to prove (iii). Clearly (iii) holds for $g=1$. We put

$$
\left(\begin{array}{l}
\varphi^{(n)}(g) \\
\psi^{(n)}(g)
\end{array}\right)=\left[\begin{array}{l}
0 ; a_{n+1}, a_{n+2}, \ldots \\
0 ; b_{n+1}, b_{n+2}, \ldots
\end{array}\right], \quad g \geq 2, n \geq 0,
$$

where $a_{n}(n \geq 3), b_{n}(n \geq 2)$ are the integers given in Theorem 1 with $a_{1}=a_{2}=b_{1}=1$. Then (6.2) implies $p<\varphi^{(0)}(g)=\varphi(g)<1,0<\psi^{(0)}(g)=$ $\psi(g)<1, n \geq 1$. In general, both $\varphi^{(n)}(g)$ and $\psi^{(n)}(g)\left(n \in \mathbb{Z}_{+}\right)$are different 
from 0 and 1 , otherwise the expansion of ${ }^{t}(\varphi(g), \psi(g))$ in the JP algorithm is terminating, which contradicts the linear independence of $1, \varphi(g)$ and $\psi(g)$ over $\mathbb{Q}$. Hence

$$
0<\varphi^{(n)}(g)=1 /\left(b_{n+1}+\psi^{(n+1)}(g)\right) \leq 1 / b_{n+1}<1, \quad n \geq 1,
$$

and therefore

$$
\begin{aligned}
0<\psi^{(n)}(g) & =\left(a_{n+1}+\varphi^{(n+1)}(g)\right) /\left(b_{n+1}+\psi^{(n+1)}(g)\right)<\left(a_{n+1}+1\right) / b_{n+1} \\
& =\left(1+g^{f_{n-2}} \sum_{m=0}^{l-1} g^{f_{n-1} m}\right) /\left(g^{l f_{n-1}} \times g^{f_{n-2}} \sum_{m=0}^{k-1} g^{f_{n} m}\right),
\end{aligned}
$$

so that $0<\psi^{(n)}(g)<1$ if $l \neq 0$ and

$$
0<\psi^{(n)}(g)=1 /\left(g^{f_{n-2}} \sum_{m=0}^{k-1} g^{f_{n} m}\right)<1
$$

if $l=0, k>1$. This implies (iii).

Proof of Theorem 2. Although the expression (6.1) is admissible in the JP algorithm when $z=g$ for any integer $g \in \mathbb{N}$, the expression (6.1) is not admissible in the JPP algorithm. Applying $T^{-1}$ and subtracting ${ }^{t}(1,1)$ from both sides of (6.1), we get

$$
\left(\begin{array}{c}
\psi(z) / \varphi(z)-1 \\
1 / \varphi(z)-1
\end{array}\right)=\left[\begin{array}{ll}
0 ; 1, & a_{3}, \ldots \\
0 ; & b_{2}, b_{3}, \ldots
\end{array}\right]
$$

Recalling that $\mathcal{A} \cup \mathcal{B} \cup \mathcal{C}(=\mathbb{N})$ is a disjoint union, we get the expression (2.9) of Theorem 2. We also get (iv) of Theorem 2 in view of (5.14) and (5.16). Next we show the admissibility of $(2.9)$. Let $\eta_{n}^{(m)}(z), \xi_{n}^{(m)}(z)$ be the functions given by

$$
\left(\begin{array}{l}
\eta_{n}^{(m)}(z) \\
\xi_{n}^{(m)}(z)
\end{array}\right)=\left[\begin{array}{l}
0 ; a_{m}, a_{m+1}, \ldots, a_{m+n} \\
0 ; b_{m}, b_{m+1}, \ldots, b_{m+n}
\end{array}\right]
$$

with $m \geq 2, n \geq 1$, and $a_{2}=1$, where $a_{n}(n \geq 3)$ and $b_{n}(n \geq 2)$ are the polynomials in $z$ in Theorem 2 . Then we have

$$
\begin{array}{ll}
\left\|\eta_{n}^{(m)}(z)\right\|=\exp \left(-\operatorname{deg} b_{m}\right) \geq e^{-1}, & m \geq 2, n \geq 1, \\
\left\|\xi_{n}^{(m)}(z)\right\|=\exp \left(\operatorname{deg} a_{m}-\operatorname{deg} b_{m}\right) \leq e^{-1}, & m \geq 2, n \geq 1,
\end{array}
$$

by using (3.4), (5.7) and the following lemma which is easily proved by induction on $n$.

Lemma 6.4 (Parusnikov [12]). Let $p_{n}, q_{n}, r_{n}(n \geq-2)$ be the polynomials given by (3.5) with $a_{n}, b_{n} \in \mathbb{C}[z](n \geq 1)$ such that $0 \leq \operatorname{deg} a_{n}<\operatorname{deg} b_{n}$. 
Then

$$
\begin{aligned}
& \operatorname{deg} p_{n}=\sum_{m=2}^{n} \operatorname{deg} b_{m}, \\
& \operatorname{deg} q_{n}=\operatorname{deg} a_{1}+\sum_{m=2}^{n} \operatorname{deg} b_{m}, \\
& \operatorname{deg} r_{n}=\sum_{m=1}^{n} \operatorname{deg} b_{m},
\end{aligned}
$$

for all $n \geq 1$.

From (6.7) we have

$$
\lim _{n \rightarrow \infty}\left\|\eta_{n}^{(m)}\right\|<1, \quad \lim _{n \rightarrow \infty}\left\|\xi_{n}^{(m)}\right\|<1, \quad m \geq 2,
$$

where the convergence follows from that of (6.1). It is clear that the above inequalities hold for $m=1$. Thus, we get the admissibility of the expression (2.9) in the JPP algorithm.

We now prove (ii). The linear independence of the functions $1, \eta(z)$, and $\xi(z)$ can be proved by the same arguments as for the numbers $1, \varphi(g)$, and $\psi(g)$, by using the inequalities corresponding to (6.7) in the case of functions in $\mathbb{K}=\mathbb{C}\left(\left(z^{-1}\right)\right)$ with metric $d_{\mathbb{K}}$ (cf. Parusnikov's theorem [12]). From this (ii) follows.

In view of Lemma $6.1, \varphi_{\mathcal{A}}(z), \varphi_{\mathcal{B}}(z)$, and $\varphi_{\mathcal{C}}(z)$ given in Theorem 2 are not rational functions, which together with the following lemma implies the transcendence result (i) in Theorem 2. The proof of Theorem 2 is complete.

LEMMA 6.5 (Carlson-Pólya). Let $f(z)$ be a function given by a power series $\sum_{n=0}^{\infty} c_{n} z^{n}\left(c_{n} \in \mathbb{Z}\right)$ with $\limsup _{n \rightarrow \infty}\left|c_{n}\right|^{1 / n}=1$. Then the unit circle $|z|=1$ is the natural boundary of $f(z)$ except when $f(z)$ is a rational function.

Proof of Theorem 3. We need the following

LEMma 6.6. Let $L=\{0,1, \ldots, g-1\}$ be the alphabet of $g$ symbols with $g \geq 2$, and let $u_{1}, u_{2}, u_{3} \in L^{+}$. Define the infinite word $v=v_{1} v_{2} \ldots v_{n} \ldots \in$ $L^{\omega}\left(v_{n} \in L\right)$ by $v=w\left(u_{1}, u_{2}, u_{3}\right)$, where $w=w(a, b, c)$ is the $\omega$-word generated by the DOL system $G$ given in Section 2. Let $M$ denote the matrix $\left(\left|u_{i}\right|_{j}\right)_{1 \leq i \leq 3,0 \leq j \leq g-1}$, where $\left|u_{i}\right|_{j}$ denotes the number defined in Theorem 3. Suppose that rank $M \geq 2$. Then $v$ is not ultimately periodic.

Proof. Put

$$
N=N(n)=\sum_{i=1}^{3}\left|\mathcal{A}_{n}^{(i)}\right| \cdot\left|u_{i}\right|,
$$


where $\mathcal{A}_{n}^{(1)}=\mathcal{A}_{n}, \mathcal{A}_{n}^{(2)}=\mathcal{B}_{n}, \mathcal{A}_{n}^{(3)}=\mathcal{C}_{n}$, which are the characteristic sets of $\sigma^{n-1}(a)$ defined by $(2.5)$, and $\left|\mathcal{A}_{n}^{(i)}\right|$ denotes the number of elements of $\mathcal{A}_{n}^{(i)}$. Then we have

Hence we get

$$
\left|v_{1} v_{2} \ldots v_{N}\right|_{j}=\sum_{i=1}^{3}\left|\mathcal{A}_{n}^{(i)}\right| \cdot\left|u_{i}\right|_{j}
$$

$$
\lim _{n \rightarrow \infty} \frac{\left|v_{1} v_{2} \ldots v_{N(n)}\right|_{j}}{N(n)}=\sum_{i=1}^{3} \alpha^{3-i}\left|u_{i}\right|_{j} / \sum_{i=1}^{3} \alpha^{3-i}\left|u_{i}\right|=\zeta_{j}, \quad \text { say },
$$

in view of Lemma 6.1. Since $\alpha$ is an irrational number of third degree, $\zeta_{j}$ is rational if and only if the vectors $\left(\left|u_{1}\right|,\left|u_{2}\right|,\left|u_{3}\right|\right)$ and $\left(\left|u_{1}\right|_{j},\left|u_{2}\right|_{j},\left|u_{3}\right|_{j}\right)$ are linearly dependent over $\mathbb{Q}$. Hence $\zeta_{j}$ is irrational for some $j(0 \leq j \leq g-1)$, since $\sum_{j=0}^{g-1}\left|u_{i}\right|_{j}=\left|u_{i}\right|$ and $\operatorname{rank} M \geq 2$. Therefore, $v$ is not ultimately periodic.

We can prove Theorem 3 by applying Lemmas 6.2 and 6.6 together with Roth's theorem, considering the rational approximation of $0 . w\left(u_{1}, u_{2}, u_{3}\right)=$ $0 . v_{1} v_{2} \ldots v_{m} \ldots$ by the rational numbers with periodic digits $0 .{ }^{*} v_{1} v_{2} \ldots \stackrel{*}{v}_{N(n)}$ $(n \geq 1)$ in their $g$-adic expansion.

As we have already seen the monoid morphism $\sigma$ defined by (2.1) has some connection with the Pisot number with minimal polynomial $x^{3}-k x^{2}-$ $l x-1$ satisfying (1.1). We can state theorems similar to Theorems 1-3 relative to the JP and JPP algorithms of dimension $s$ and Pisot numbers with minimal polynomial $x^{s-1}-k_{s} x^{s}-k_{s-1} x^{s-1}-\ldots-k_{1} x-1$ under the conditions $1 \leq k_{1} \leq k_{2} \leq \ldots \leq k_{s}\left(k_{i} \in \mathbb{Z}\right)$ for any given $s \geq 1$. We will give such results in forthcoming papers.

\section{References}

[1] W. W. Adams and J. L. Davison, A remarkable class of continued fractions, Proc. Amer. Math. Soc. 65 (1977), 194-198.

[2] P. E. Böhmer, Über die Transzendenz gewisser dyadischer Brüche, Math. Ann. 96 (1927), 367-377; Berichtigung, ibid. 735.

[3] P. Bundschuh, Über eine Klasse reeller transzendenter Zahlen mit explizit angebbarer g-adischer und Kettenbruch-Entwicklung, J. Reine Angew. Math. 318 (1980), $110-119$.

[4] L. V. Danilov, Some classes of transcendental numbers, Mat. Zametki 12 (2) (1972), 149-154 (in Russian); English transl.: Math. Notes 12 (1972), 524-527.

[5] J. L. Davison, A series and its associated continued fraction, Proc. Amer. Math. Soc. 63 (1977), 29-32.

[6] J. H. Loxton and A. J. van der Poorten, Arithmetic properties of certain functions in several variables, III, Bull. Austral. Math. Soc. 16 (1977), 15-47. 
[7] K. Mahler, Arithmetische Eigenschaften der Lösungen einer Klasse von Funktionalgleichungen, Math. Ann. 101 (1929), 342-366.

[8] D. Masser, A vanishing theorem for power series, Invent. Math. 67 (1982), 275296.

[9] E. M. Nikishin and V. N. Sorokin, Rational Approximations and Orthogonality, Nauka, Moscow 1988, 168-175 (in Russian).

[10] K. Nishioka, Evertse theorem in algebraic independence, Arch. Math. (Basel) 53 (1989), 159-170.

[11] K. Nishioka, I. Shiokawa and J. Tamura, Arithmetical properties of certain power series, J. Number Theory, to appear.

[12] V. I. Parusnikov, The Jacobi-Perron algorithm and simultaneous approximation of functions, Mat. Sb. 114 (156) (2) (1981), 322-333 (in Russian).

[13] A. Salomaa, Jewels of Formal Language Theory, Pitman, 1981.

[14] —, Computation and Automata, Cambridge Univ. Press, 1985.

[15] J. Tamura, Transcendental numbers having explicit g-adic and Jacobi-Perron expansions, in: Séminaire de Théorie des Nombres de Bordeaux, to appear.

FACULTY OF GENERAL EDUCATION

INTERNATIONAL JUNIOR COLLEGE

EKODA 4-15-1, NAKANO-KU

TOKYO 165, JAPAN 